\title{
Collaborative Care for Depression and Posttraumatic Stress Disorder: Evaluation of Collaborative Care Fidelity on Symptom Trajectories and Outcomes
}

\author{
Bradley E. Belsher, $P h D^{1,2}$, Daniel P. Evatt, $P h D^{1,2}$, Xian Liu, PhD ${ }^{1,2}$, Michael C. Freed, PhD ${ }^{2,3}$, \\ Charles C. Engel, MD, MPH ${ }^{2,4}$, Erin H. Beech, MA', and Lisa H. Jaycox, $P h D^{4}$ \\ 'Psychological Health Center of Excellence, Defense Health Agency, Silver Spring, MD, USA; ${ }^{2}$ Uniformed Services University of the Health Sciences, \\ Bethesda, MD, USA; ${ }^{3}$ Division of Services and Intervention Research, National Institute of Mental Health (NIMH), Bethesda, MD, USA; ${ }^{4}$ RAND \\ Corporation, Arlington, VA, USA.
}

\begin{abstract}
BACKGROUND: Despite the growing consensus that collaborative care is effective, limited research has focused on the importance of collaborative care fidelity as it relates to mental health clinical outcomes.

OBJECTIVE: To assess the relationship of collaborative care fidelity on symptom trajectories and clinical outcomes among military service members enrolled in a multi-site randomized controlled trial for the treatment of depression and posttraumatic stress disorder (PTSD). DESIGN: Study data for our analyses came from a twoparallel arm randomized trial that evaluated the effectiveness of a centralized collaborative care model compared to the existing collaborative care model for the treatment of PTSD and depression. All patients were included in the analyses to evaluate how longitudinal trajectories of PTSD and depression scores differed across various collaborative care fidelity groupings.
\end{abstract}

PARTICIPANTS: A total of 666 US Military Service members screening positive for probable PTSD or depression through primary care.

MAIN MEASURES: Disease registry data from a webbased clinical management support tool was used to measure collaborative care fidelity for patients enrolled in the trial. Participant depression and PTSD symptoms were collected independently from research survey assessments at four time points across the 1-year trial period. Treatment utilization records were acquired from the Military Health System administrative records to determine mental health service use.

KEY RESULTS: Consistent and late fidelity to the collaborative care model predicted an improving symptom trajectory over the course of treatment. This effect was more pronounced for patients with depression than for patients with PTSD.

CONCLUSIONS: Long-term fidelity to key collaborative care elements throughout care episodes may improve depression outcomes, particularly for patients with elevated symptoms. More controlled research is needed to further understand the influence of collaborative care fidelity on clinical outcomes.

TRIAL REGISTRATION: Clinicaltrials.gov Identifier NCT01492348

Received September 15, 2017

Revised February 13, 2018

Accepted March 28, 2018

Published online April 27, 2018
KEY WORDS: posttraumatic stress disorder; depression; collaborative care; treatment fidelity.

J Gen Intern Med 33(7):1124-30

DOI: $10.1007 / \mathrm{s} 11606-018-4451-5$

(c) Society of General Internal Medicine (This is a U.S. Government work and not under copyright protection in the US; foreign copyright protection may apply) 2018

$G$ rounded in the chronic care model, ${ }^{1}$ collaborative care is a population-based care approach designed to improve the management of patients treated in the primary care setting. Collaborative care is composed of a package of core elements that facilitate long-term patient engagement and management: a prepared primary care practice with assigned provider roles and procedures, care managers who routinely contact patients, regular administration of validated symptom scales to track patient clinical trajectories, and ongoing consultation with behavioral health specialists who can recommend treatment changes for patients as needed. ${ }^{2,} 3$ Essential to the model, a web-based clinical management support tool allows longitudinal symptom tracking of patients, communication between team members, and documentation of patient treatment history including indications that a patient may be lost to care. ${ }^{4}$

Collaborative care is an effective treatment across a range of mental health disorders and comorbidities, ${ }^{5}$ and several large health care systems have implemented the care model. ${ }^{6} \mathrm{Re}$ cently, the Center for Medicare and Medicaid Services (CMS) updated payment policies to include monthly reimbursement for Collaborative Care Management (CoCM) services for Medicare beneficiaries. ${ }^{7,8}$ These codes allow Medicare to be billed when a threshold amount of time is spent during a month delivering required $\mathrm{CoCM}$ elements that include ongoing care management (remotely or in-person), administration of validated measures, tracking of patient symptoms using a disease registry, and psychiatric consultation. ${ }^{9}, 10$

Despite the growing consensus that collaborative care is effective and national efforts to disseminate the model into standard practice, limited research has focused on the importance of 
collaborative care fidelity as it relates to clinical outcomes. A meta-analysis on collaborative care for depression found that higher fidelity to collaborative care elements was associated with higher pooled effect sizes relative to studies with lower fidelity, whereas the addition of specific psychotherapy or initiation of antidepressants at the start of treatment was not associated with significantly increased effect size. ${ }^{11}$ Thus, the consistent delivery of central collaborative care components may be important to optimize the impact of the care model. Notably, Oxman et al. ${ }^{12}$ developed and tested a collaborative care fidelity measure based on stakeholder ratings and using care manager logs to predict outcomes among participants enrolled in a collaborative care trial. Although this measure demonstrated that fidelity predicted improved treatment outcomes, the tool included items that already measured patient improvement, required a complex weighting algorithm, and was not used to analyze longitudinal trajectories of patients. Therefore, further research is needed to examine the effects of CoCM fidelity on clinical outcomes.

Engel et al. ${ }^{13}$ tested the comparative effectiveness of two collaborative care models for posttraumatic stress disorder (PTSD) and depression among military service members treated in the Military Health System (MHS) primary care setting. Although the care models differed in important ways (i.e., usual collaborative care versus an enhanced collaborative care with stepped treatment options), the essential collaborative care elements previously described were the main components of both care models. The current research seeks to evaluate data from this trial to enhance our understanding of how CoCM services affect symptom trajectories and clinical outcomes among patients with depression and PTSD across their year-long period of care. To examine the role of $\mathrm{CoCM}$, we analyzed the longitudinal effect of four distinct CoCM fidelity patterns on clinical trajectories, predicting that increased fidelity would be associated with improved clinical outcomes.

\section{METHODS}

The study was reviewed and approved by institutional review boards at Walter Reed National Military Medical Center, six participating Army installations, RTI International, RAND Corporation, University of Washington, the Boston VA, and the Human Research Protection Office, US Army Medical Research and Materiel Command. Written informed consent was obtained after full explanation of study procedures.

\section{Design}

Study data came from a two-parallel arm randomized trial that evaluated the effectiveness of a centralized collaborative care model compared to the existing collaborative care model for US Military Service members screening positive for probable PTSD or depression through primary care. Full details on the design and outcomes of the randomized trial are available elsewhere. ${ }^{13-15}$ All patients across both treatment arms were included in the current analyses.

\section{Participants}

Active duty military service members were recruited from six large military posts (18 primary care clinics across all six sites) across the USA from 2012 to 2015, with data collected from February 2012 through August 2016. Eligible participants met DSM IV-TR ${ }^{16}$ criteria for probable PTSD on the PTSD Checklist-Civilian Version (PCL-C; a "moderate" or greater severity level on 1 re-experiencing, 3 avoidance, and 2 hyperarousal symptoms $)^{17}$ and/or probable depression on the Patient Health Questionnaire-9 (PHQ-9; endorsement of at least 5 of the 9 symptoms experienced "more than half the days" and at least 1 of those symptoms must include either "little interest or pleasure in doing things" or "feeling down, depressed or hopeless"), and reported having Internet and e-mail access. ${ }^{18}$ Participants were excluded for current alcohol dependence or active suicidal ideation.

\section{Interventions}

Both collaborative care interventions were based on the "three component model" ${ }^{3}$ that encompasses (1) primary care-based management to include uniform screening and referral, symptom measurement, and care management support; (2) regular psychiatric consultation to inform treatment planning; and (3) use of a web-based clinical management support tool and disease registry. The enhanced model also included additional telehealth treatment options and a centralized team that monitored care processes and provided centralized psychiatric consultation to care managers to improve treatment sequencing. Notably, the two treatment interventions varied in their emphasis on treatment engagement. Therefore, to minimize the potential influence of this confounding effect, treatment arm and mental health treatment utilization were included as control variables in the current analyses.

\section{Measures}

Mental Health Outcome Measures. The study outcome measures were independently collected via computer administration at baseline, 3 months, 6 months, and 12 months. Depression symptom severity was assessed using the Hopkins Symptom Checklist Depression Scale-20 Item Version (HSCL-20), ${ }^{19}$ a 20 -item self-report scale that includes all diagnostic symptoms of major depression and demonstrates strong sensitivity to clinical change. PTSD was assessed using the Posttraumatic Diagnostic Scale (PDS), ${ }^{20}$ a 49-item self-report measure to assess the severity of PTSD symptoms related to an identified traumatic event.

Collaborative Care Management Fidelity. Data was derived from a web-based care manager support tool, standardly used in both care models, to measure CoCM fidelity. The support tool tracked care manager contacts, administration of symptom measures, and consultation with mental health specialists 
across both arms of the study. Data was extracted at the patient level for the 1-year period of enrollment in the study to evaluate CoCM fidelity throughout the patients' year period of care. Based on the central components of collaborative care, ${ }^{21}$ we defined collaborative care management as the presence of the following required elements within the same month period: a care management contact with the patient (remote or in-person), symptom monitoring using a validated scale with entry of scores into a disease registry, and psychiatric consultation between the care manager and the behavioral health specialist (Table 1). CoCM was measured as a timevarying, dichotomous variable, coded as present if all the defined collaborative care management elements were recorded within the same month.

The results of a preliminary analysis indicated that participants with at least 2 months of CoCM at the beginning of care reported significantly greater symptoms earlier in care relative to those with one or fewer months of CoCM, whereas those with at least 2 months of CoCM during the last period of care reported significantly lower symptoms by the end of treatment ( $p=.003$ ). Based on these observed clustering patterns, CoCM fidelity was categorized into four mutually exclusive groups: high fidelity if the participant received two or more months of CoCM during both six-month periods of care; early fidelity if the participant received two or more months of CoCM in the first six, but not the last six months of care; late fidelity if the participant received two or more months of CoCM in the last six, but not first six months of care; and low fidelity if the participant received less than 2 months of CoCM in the entire year of the study period.

Treatment Utilization. Individual-level claims and encounter data were acquired directly from the MHS Medical Data Repository (MDR). The MDR includes the official utilization records of all health care visits for both direct and purchased care for all service members. The data was extracted at the patient level for the 1-year period the participant was enrolled in the study. Mental health specialty care encounters were summed for each participant across the year period. Pharmacy records were used to determine whether participants received any antidepressant medications throughout the trial. Participants were considered engaged in care if they either received at least 4 mental health specialty care encounters or were prescribed an antidepressant medication, within 3-month timeframes across the trial. This allowed us to control for treatment engagement as a time-varying factor across patients' care,

Table 1 Collaborative Care Management (CoCM) Definition

All elements occurring within the same month timeframe:

1. Care management contact with patient (remotely or in-person)

2. Symptom monitoring using a validated measure and entry of symptom scores into a disease registry

3. Psychiatric consultation

Based on the Center for Medicare and Medicaid Services payment policies for Collaborative Care Management (CoCM) services for Medicare beneficiaries thus better accounting for any potential confounding effects of treatment utilization on clinical outcomes.

Psychosocial Characteristics. Demographic variables were collected at baseline using validated survey items. Healthrelated quality of life was assessed using the Short Form-12 (SF-12), ${ }^{22}$ with two subscales measuring physical (Physical Component Scale; PCS) and mental (Mental Component Scale; MCS) related functioning. The MCS was used as a control variable in the analyses to address any potential confounding effects of differences in mental health functioning between the groups at baseline.

\section{Analysis}

We examined the effect of CoCM fidelity group on clinical trajectories across all four time points, controlling for treatment arm, age, gender, baseline mental health functioning, and time-varying treatment engagement. For all analyses, we used a linear mixed model to address intra-individual correlation and adjust for case and item missing data in the analyses. ${ }^{23}$ The restricted maximum likelihood estimator was used to find unbiased parameter estimates. The time factor consisted of two components, time and time $\times$ time, for capturing nonlinearity of longitudinal trajectories. The individual-specific random effects were specified for the intercept and for the slope of time. The SAS PROC MIXED procedure was used to compute both the fixed and the random effects for the linear mixed model. ${ }^{24-26}$ The analyses were conducted at each level of patient observation and included all participants randomized into the trial.

\section{RESULTS}

Psychosocial and care process characteristics across the four fidelity groups are reported in Table 2 . Baseline characteristics across the groups appear relatively similar. Care process characteristics differed considerably, with low and high fidelity groups receiving the least and most collaborative care elements, respectively, and the early and late fidelity groups receiving roughly similar amounts of care. Based on a previous meta-analysis, the range of care manager contacts across the entire sample appears comparable to other collaborative care studies. $^{27}$ Table 3 reports on the mental health treatment utilization patterns for each group across care periods. The trends indicate that the high fidelity group tended to have the highest proportion of patients engaged in mental health treatments throughout the year, slightly declining during the last 3 months. The other three groups appear to have similar levels of treatment engagement during the first 6 months of care. However, the late fidelity group continued to experience relatively higher levels of treatment engagement during the last 6 months, whereas the early and low fidelity groups experience declining levels of treatment. These observed treatment 
Table 2 Characteristics across Collaborative Care Management (CoCM) Groups

\begin{tabular}{|c|c|c|c|c|c|}
\hline & $\begin{array}{l}\text { Low fidelity } \\
(n=397)\end{array}$ & $\begin{array}{l}\text { Early fidelity } \\
(n=109)\end{array}$ & $\begin{array}{l}\text { Late fidelity } \\
(n=47)\end{array}$ & $\begin{array}{l}\text { High fidelity } \\
(n=113)\end{array}$ & $\begin{array}{l}\text { Total sample } \\
(N=666)\end{array}$ \\
\hline \multicolumn{6}{|l|}{ Demographics } \\
\hline Age, years (SD) & $31.03(7.4)$ & $30.47(7.98)$ & $31.64(8.23)$ & $31.72(8.0)$ & $31.44(7.66)$ \\
\hline Male gender, $n(\%)$ & $329(82.9 \%)$ & $88(80.7 \%)$ & $36(76.6 \%)$ & $86(76.1 \%)$ & $539(80.9 \%)$ \\
\hline Caucasian Race, $n(\%)$ & $197(49.6 \%)$ & $52(47.7 \%)$ & $22(46.8 \%)$ & $46(40.7 \%)$ & $317(47.6 \%)$ \\
\hline High school degree, $n(\%)$ & $271(68.3 \%)$ & $77(70.6 \%)$ & $35(74.5 \%)$ & $80(70.8 \%)$ & $463(69.5 \%)$ \\
\hline Married, $n(\%)$ & $245(61.7 \%)$ & $69(63.3 \%)$ & $31(66.0 \%)$ & $72(63.7 \%)$ & $417(62.6 \%)$ \\
\hline \multicolumn{6}{|l|}{ Baseline symptoms } \\
\hline PTSD Severity ${ }^{\mathrm{a}}$, mean (SD) & $28.59(9.00)$ & $29.63(9.15)$ & $29.77(10.99)$ & $30.46(8.92)$ & $29.16(9.17)$ \\
\hline Depression Severity $^{\mathrm{b}}$, mean (SD) & $14.67(4.60)$ & $15.18(4.65)$ & $16.06(3.86)$ & $15.28(4.52)$ & $14.95(4.55)$ \\
\hline Physical Functioning ${ }^{\mathrm{c}}$, mean (SD) & $39.64(11.58)$ & $40.02(11.14)$ & $40.18(10.96)$ & $38.97(10.40)$ & $39.63(11.26)$ \\
\hline Mental Functioning ${ }^{\mathrm{c}}$, mean (SD) & $32.18(10.23)$ & $31.83(9.85)$ & $29.61(9.32)$ & $31.46(8.32)$ & $31.82(9.81)$ \\
\hline \multicolumn{6}{|l|}{ CoCM care processes across the 12 months of care } \\
\hline Month of care manager contacts, mean (SD) & $3.43(3.01)$ & $5.20(2.10)$ & $6.74(2.10)$ & $8.01(1.99)$ & $4.73(3.20)$ \\
\hline Months of symptom assessments, mean (SD) & $3.10(2.88)$ & $4.88(1.93)$ & $6.23(1.97)$ & $7.74(1.90)$ & $4.40(3.10)$ \\
\hline Months of psychiatric consultation, mean (SD) & $2.10(2.13)$ & $6.36(2.72)$ & $6.15(2.26)$ & $8.74(1.94)$ & $4.21(3.47)$ \\
\hline Months of CoCM, mean (SD) & $0.55(0.68)$ & $2.95(1.10)$ & $3.40(1.01)$ & $6.28(1.75)$ & $2.12(2.39)$ \\
\hline
\end{tabular}

${ }^{a}$ Based on the Posttraumatic Diagnostic Scale (PDS)

${ }^{b}$ Based on the Patient Health Questionnaire-9 (PHQ-9)

${ }^{c}$ Based on the Short Form-12 Physical Component Scale (PCS) and Mental Component Scale (MCS)

patterns underscored the importance of using treatment engagement as a time-varying control variable in our analyses.

For the primary analyses, all random effects were statistically significant $(p<0.001)$, thereby indicating the importance of applying the linear mixed model on the longitudinal data. The first analysis indicated a statistically significant interaction between time and $\mathrm{CoCM}$ group on depression symptoms $(F(\mathrm{DF}$ Num $=3$, DF Den $=1787)=3.06, p=0.027)$, controlling for mental health functioning, treatment engagement, and other confounders. In Figure 1, the predicted longitudinal trajectories of depressive symptom scores for the four CoCM groups adjusting for the potential confounding effects are presented. As illustrated, symptom trajectories of the high and late fidelity groups indicated slightly higher depressive symptoms at baseline with significantly sharper declines in symptoms relative to the other groups during the last 6 months of care. We next analyzed the effect of long-term fidelity on PTSD outcomes. There were no statistically significant differences in symptom trajectories among the groups $(p<0.156)$ despite similar patterns (Fig. 2).

\section{DISCUSSION}

Fidelity to core collaborative care components demonstrated differential effects on clinical trajectories. For depression, long-term and later fidelity were both associated with significantly greater decreases in depressive symptoms relative to early and low fidelity groups. Symptom trajectories among all four groups appeared to decline steadily in the first 6 months of care. However, the low and early fidelity trajectories plateaued during the last 6 months of care, whereas the high and late fidelity groups continued to experience declining symptoms.

Treatment utilization was controlled for in the analyses, and accordingly the distinct symptom improvements between the groups cannot be solely attributed to greater mental health treatment receipt. However, the potential effects of treatment utilization, in addition to fidelity, on treatment outcomes cannot be ruled out. Although all groups received seemingly similar levels of antidepressant or psychotherapy treatment within the first 6 months of care, the longer-term and later fidelity groups appeared more likely to receive treatment during the last period of care, particularly the last 3 months.

Table 3 Treatment Utilization Across Time and Collaborative Care Management (CoCM) Groups

\begin{tabular}{|c|c|c|c|c|}
\hline & $\begin{array}{l}1 \text { to } 3 \text { months } \\
\text { into care }\end{array}$ & $\begin{array}{l}4 \text { to } 6 \text { months } \\
\text { into care }\end{array}$ & $\begin{array}{l}7 \text { to } 9 \text { months } \\
\text { into care }\end{array}$ & $\begin{array}{l}10 \text { to } 12 \text { months } \\
\text { into care }\end{array}$ \\
\hline \multicolumn{5}{|c|}{ Patients receiving $>4$ behavioral health encounters/period, number $(\%)$} \\
\hline Low fidelity & $142(35.8 \%)$ & $101(25.4 \%)$ & $79(19.9 \%)$ & $61(15.4 \%)$ \\
\hline Early fidelity & $36(33.0 \%)$ & $29(26.6 \%)$ & $22(20.2 \%)$ & $12(11.0 \%)$ \\
\hline Late fidelity & $17(36.2 \%)$ & $13(27.7 \%)$ & $11(23.4 \%)$ & $12(25.5 \%)$ \\
\hline High fidelity & $51(45.1 \%)$ & $50(44.2 \%)$ & $41(36.3 \%)$ & $29(25.7 \%)$ \\
\hline \multicolumn{5}{|c|}{ Patients receiving antidepressant medication/period, number $(\%)$} \\
\hline Low fidelity & $210(52.9 \%)$ & $169(42.6 \%)$ & $154(38.8 \%)$ & $135(34.0 \%)$ \\
\hline Early fidelity & $70(64.2 \%)$ & $50(45.9 \%)$ & $41(37.6 \%)$ & $38(34.9 \%)$ \\
\hline Late fidelity & $29(61.7 \%)$ & $19(40.4 \%)$ & $23(48.9 \%)$ & $23(48.9 \%)$ \\
\hline High fidelity & $79(69.9 \%)$ & $77(68.1 \%)$ & $74(65.5 \%)$ & $63(55.8 \%)$ \\
\hline \multicolumn{5}{|c|}{ Patients receiving $\geq 4$ behavioral health encounters or antidepressant medication/period, number $(\%)$} \\
\hline Low fidelity & $248(62.5 \%)$ & $199(50.1 \%)$ & $173(43.6 \%)$ & $148(37.3 \%)$ \\
\hline Early fidelity & $76(69.7 \%)$ & $62(56.9 \%)$ & $48(44.0 \%)$ & $40(36.7 \%)$ \\
\hline Late Fidelity & $32(68.1 \%)$ & $24(51.1 \%)$ & $24(51.1 \%)$ & $27(57.4 \%)$ \\
\hline High fidelity & $89(78.8 \%)$ & $86(76.1 \%)$ & $80(70.8 \%)$ & $67(59.3 \%)$ \\
\hline
\end{tabular}




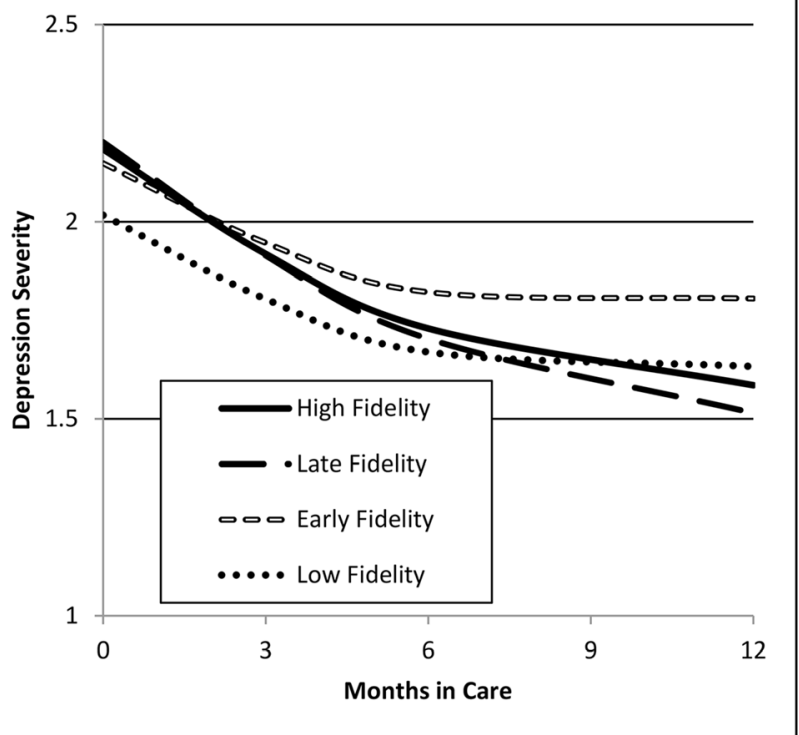

Figure 1 Longitudinal clinical trajectories of depression severity (HSCL $\dagger$ ) for 4 collaborative care management (CoCM) fidelity groups $(N=666)$. + HSCL: Hopkins Symptom Checklist Depression Scale-20 Item Version. - Low Fidelity: less than 2 months of collaborative care management services in the first and last six months of care. - Early Fidelity: two or more months of collaborative care management services in the first six, but not last six months of care. - Late Fidelity: two or more months of collaborative care management services in the last six, but not first six months of care. - High Fidelity: two or more months of collaborative care management services in the first and last six months of care.

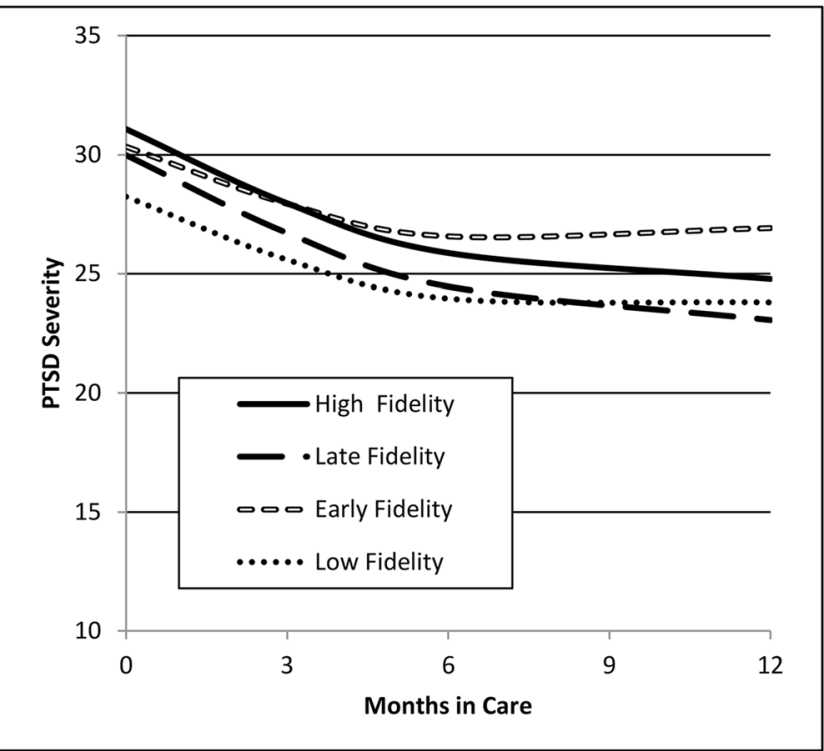

Figure 2 Longitudinal clinical trajectories of PTSD severity (PDS $\uparrow)$ for 4 collaborative care management $(\mathrm{CoCM})$ fidelity groups $(N=$ 666). $\uparrow$ PDS: Posttraumatic Diagnostic Scale. $\bullet$ Low Fidelity: less than 2 months of collaborative care management services in the first and last six months of care. $•$ Early Fidelity: two or more months of collaborative care management services in the first six, but not last six months of care. - Late Fidelity: two or more months of collaborative care management services in the last six, but not first six months of care. - High Fidelity: two or more months of collaborative care management services in the first and last six months of care.
This might suggest that whereas the majority of patients enrolling into a collaborative care intervention at the beginning of care may already be largely receptive to and engaged in treatment, longer-term CoCM may enable patients who drop from care earlier on to re-engage in care at a later point. The interactive effects of care fidelity and treatment engagement on clinical outcomes should be further explored in future research.

It is noteworthy that although the long-term and late fidelity groups were associated with greater depression symptom reductions relative to the other groups, their post-treatment depression symptoms were largely equal to the low fidelity group. Thus, some of the differential effect of care fidelity on symptom trajectories may reflect the tailored nature of collaborative care and its emphasis on treating patients based on symptom severity. Patients receiving higher levels of care may be in greater distress at that point of care and therefore may be more interested in the care offered, whereas other patients may not require intensive or long-term CoCM. Alternatively, care managers may put in more effort to engage patients that are known to have the highest need.

The same effect of long-term fidelity was not observed when PTSD was the primary outcome. Although symptom patterns looked similar, long-term and late fidelity were not associated with significantly greater PTSD reductions relative to the other groups. Notably, two recent trials on collaborative care for PTSD showed no effect on PTSD outcomes. ${ }^{28,29}$ The lack of significant findings regarding care fidelity on PTSD outcomes may suggest that active implementation and availability of evidence-based treatments for PTSD may be a necessary prerequisite to effect changes on PTSD beyond the presence of a collaborative framework. ${ }^{30}$ Specifically, in two collaborative care trials for PTSD, participants receiving collaborative care had better access to care and received higher quality care, but collaborative care did not confer clinical benefit on PTSD symptoms. ${ }^{28,}{ }^{29}$ However, a third collaborative care trial that explicitly added an evidence-based, manualized PTSD treatment into the care approach demonstrated significant PTSD symptom improvements as compared to usual care. ${ }^{31}$ Although collaborative care fidelity may serve to increase access to treatments and provide a safety net for those patients who do not respond to care, specific evidencebased PTSD treatments likely need to be actively implemented and available within the care model.

Overall, the results are generally consistent with other research documenting the importance of collaborative care fidelity on symptom outcomes. ${ }^{32}$ Extending previous findings that suggest that patients who engage early in collaborative care tend to benefit more, ${ }^{33-36}$ our results indicate that early engagement alone may not be sufficient for patients if they subsequently drop out of care. The goal of collaborative care to facilitate long-term patient engagement and management may be especially important given the high rates of mental health treatment drop-out. ${ }^{37}$ Finally, while a previous meta-analysis did not find a relationship between care management sessions 
and clinical outcomes, ${ }^{27}$ our results suggest that CoCM fidelity may provide a more useful predictor of depression outcomes.

There are several limitations to the current study. First, data was observational and levels of collaborative care were not controlled across participants. Consistent CoCM may be associated with other participant and care characteristics such as motivation and receptivity to care, not measured here, and greater adherence to treatments. ${ }^{28}$ Although the different collaborative care interventions may have emphasized care fidelity and engagement to different degrees, the effect of treatment arm and treatment engagement was controlled for in all analyses, statistically adjusting for the confounding effect of the intervention on the analyses. Finally, as this is the first study we are aware of to apply the CoCM definition to measure collaborative care fidelity on long-term clinical outcomes, it is unclear how well CoCM represents true collaborative care fidelity. There may be more nuanced ways to measure collaborative care fidelity and its effect on clinical trajectories. However, based on recent policy and CMS reimbursement decisions, CoCM may be a meaningful metric to monitor across large health systems to promote value-based care. ${ }^{32}$ Further research is needed to examine the validity and utility of this construct.

In conclusion, this research sought to advance knowledge on the implementation and sustainment of collaborative care by evaluating the effect of CoCM fidelity on long-term clinical outcomes. Our results suggest that long-term and late collaborative care engagement for depression may play an important factor in clinical outcomes by potentially ensuring that patients remain engaged in care throughout care episodes. More controlled research to inform the optimal sequencing and intensity of collaborative care delivery for target populations is warranted.

Acknowledgements: We wish to thank the committed care managers, research site investigators, site coordinators, primary care clinicians, clinic staff, and mental health specialists who supported the STEPS-UP study.

The STEPS-UP study was supported by a Department of Defense Deployment Related Medical Research Program award (Grant DR080409). The award was a joint award to the Henry M. Jackson Foundation for the Advancement of Military Medicine, Inc. (award W81XWH-09-2-0077), Research Triangle Institute (award W81XWH09-2-0078), and RAND Corporation (award W81XWH-09-2-0079). The sponsor had no role in study design; in the collection, analysis and interpretation of data; in the writing of the report; or in the decision to submit the article for publication. The views expressed in this article are those of the authors and do not necessarily represent the views of the Department of Veterans Affairs, the Department of Defense, the National Institutes of Health, or any US government agency.

Corresponding Author: Bradley E. Belsher, PhD; Psychological Health Center of Excellence, Defense Health Agency, Silver Spring, MD, USA (e-mail: bradley.e.belsher.civ@mail.mil).

Compliance with Ethical Standards: The study was reviewed and approved by institutional review boards at Walter Reed National Military Medical Center, six participating Army installations, RTI International, RAND Corporation, University of Washington, the Boston VA, and the Human Research Protection Office, US Army Medical Research and Materiel Command. Written informed consent was obtained after full explanation of study procedures.
Conflict of Interest: The authors declare that they do not have a conflict of interest.

\section{REFERENCES}

1. Wagner EH, Austin BT, Von Korff M. Organizing care for patients with chronic illness. Milbank Q. 1996;74(4):511-44.

2. The University of Washington's AIMS Center. Collaborative care. Available at: https://aims.uw.edu/collaborative-care. Accessed February 22, 2018.

3. Oxman TE, Dietrich AJ, Williams JW, Kroenke K. A three-component model for reengineering systems for the treatment of depression in primary care. Psychosomatics. 2002;43(6):441-50.

4. Unützer J, Choi Y, Cook IA, Oishi S. Clinical computing: a web-based data management system to improve care for depression in a multicenter clinical trial. Psychiatr Serv. 2002;53(6):671-8.

5. Archer J, Bower P, Gilbody S, et al. Collaborative care for depression and anxiety problems. Cochrane Database Syst Rev. 2012;10:CD006525.

6. Katon W, Unützer J, Wells $\mathbf{K}$, Jones L. Collaborative depression care: history, evolution and ways to enhance dissemination and sustainability. Gen Hosp Psychiatry. 2010;32(5):456-464.

7. Katon WJ, Unützer $\mathbf{J}$. Health reform and the Affordable Care Act: the importance of mental health treatment to achieving the triple aim. J Psychosom Res. 2013;74(6):533-7.

8. Unützer J, Harbin H, Schoenbaum M, Druss, B. The collaborative care model: an approach for integrating physical and mental health care in medicaid health homes. Available at: https://aims.uw.edu/sites/default/files/CMSBrief_2013.pdf. Accessed 22 Feb 2018.

9. Federal Register. Medicare program; revisions to payment policies under the physician fee schedule and other revisions to Part B for CY 2017; medicare advantage bid pricing data release; medicare advantage and Part D medical loss ratio data release; medicare advantage provider network requirements; expansion of medicare diabetes prevention program model; medicare shared savings program requirements. Available at: https://www.gpo.gov/fdsys/pkg/FR-2016-07-15/pdf/2016-16097. pdf. Accessed 22 Feb 2018.

10. Press MJ, Howe R, Schoenbaum M, et al. Medicare payment for behavioral health integration. N Engl J Med. 2017;376(5):405-7.

11. Gilbody S, Bower P, Fletcher J, Richards D, Sutton AJ. Collaborative care for depression: a cumulative meta-analysis and review of longer-term outcomes. Arch Intern Med. 2006;166(21):2314-21.

12. Oxman TE, Schulberg HC, Greenberg RL, et al. A fidelity measure for integrated management of depression in primary care. Med Care. 2006;44(11):1030-7.

13. Engel CC, Bray RM, Jaycox LH, et al. Implementing collaborative primary care for depression and posttraumatic stress disorder: design and sample for a randomized trial in the U.S. Military Health System. Contemp Clin Trials. 2014;39(2):310-9.

14. Belsher BE, Jaycox LH, Freed MC, et al. Mental health utilization patterns during a stepped, collaborative care effectiveness trial for PTSD and depression in the military health system. Med Care. 2016:54(7):706-13.

15. Engel CC, Jaycox LH, Freed MC, et al. Centrally assisted collaborative telecare for posttraumatic stress disorder and depression among military personnel attending primary care: a randomized clinical trial. JAMA Intern Med. 2016;176(7):948-56.

16. American Psychiatric Association. Diagnostic and Statistical Manual of Mental Disorders. $4^{\text {th }}$ ed., text revision. Washington, DC, American Psychiatric Association Publishing; 2000:467-8.

17. Weathers FW, Litz BT, Herman JA, Huska JA, Keane TM. The PTSD Checklist (PCL): reliability, validity, and diagnostic utility. Paper presented at: ISTSS 1993. Proceedings of the $9^{\text {th }}$ Annual Convention of the International Society for Traumatic Stress Studies; 1993;San Antonio, TX.

18. Spitzer RL, Kroenke K, Williams JB. Validation and utility of a selfreport version of PRIME-MD: the PHQ primary care study. JAMA. 1999;282(18):1737-44.

19. Williams JW Jr, Stellato CP, Cornell J, Barrett JE. The 13-and 20-item Hopkins Symptom Checklist Depression Scale: psychometric properties in primary care patients with minor depression or dysthymia. Int $\mathrm{J}$ Psychiatry Med. 2004;34(1):37-50.

20. Foa EB, Cashman L, Jaycox L, Perry K. The validation of a self-report measure of posttraumatic stress disorder: the Posttraumatic Diagnostic Scale. Psychol Assessment. 1997;9(4):445-51.

21. Katon WJ, Seeling $\mathbf{M}$. Population-based care of depression: team care approaches to improving outcomes. J Occup Environ Med. 2008;50(4):459-67 
22. Ware J Jr, Kosinski M, Keller SD. A 12-Item Short-Form Health Survey: construction of scales and preliminary tests of reliability and validity. Med Care. 1996;34(3):220-33.

23. Fitzmaurice G, Laird N, Ware J. editors. Applied Longitudinal Analysis. New Jersey: John Wiley \& Sons; 2004.

24. Littell RC, Milliken GA, Stroup WW, Wolfinger RD, Schabenberger 0. SAS for Mixed Models. 2nd North Carolina: SAS Institute; 2006.

25. Liu X. Methods and Applications of Longitudinal Data Analysis. New York: Academic Press; 2015.

26. SAS Institute Inc. SAS/STAT® 12.1 User's Guide. North Carolina: SAS Institute; 2012

27. Bower P, Gilbody S, Richards D, Fletcher J, Sutton A. Collaborative care for depression in primary care. Br J Psychiatry. 2006;189:484-93.

28. Meredith LS, Eisenman DP, Han B, et al. Impact of collaborative care for underserved patients with PTSD in primary care: a randomized controlled trial. J Gen Intern Med. 2016;31(5):509-17.

29. Schnurr PP, Friedman MJ, Oxman TE, et al. RESPECT-PTSD: reengineering systems for the primary care treatment of PTSD, a randomized controlled trial. J Gen Intern Med. 2013;28(1):32-40.

30. Rosen CS, Matthieu MM, Wiltsey Stirman S, et al. A review of studies on the system-wide implementation of evidence-based psychotherapies for posttraumatic stress disorder in the Veterans Health Administration. Adm Policy Ment Health. 2016;43(6):957-77.
31. Fortney JC, Pyne JM, Kimbrell TA, et al. Telemedicine-based collaborative care for posttraumatic stress disorder: a randomized clinical trial. JAMA Psychiatry. 2015;72(1):58-67.

32. Bao Y, McGuire TG, Chan YF, et al. Value-based payment in implementing evidence-based care: the Mental Health Integration Program in Washington State. Am J Manag Care. 2017;23(1):48-53.

33. Bauer AM, Azzone V, Goldman HH, et al. Implementation of collaborative depression management at community-based primary care clinics: an evaluation. Psychiatr Serv. 2011;62(9):1047-53.

34. Bodenheimer $\mathbf{T}$, Lorig $\mathbf{K}$, Holman $\mathbf{H}$, Grumbach $\mathbf{K}$. Patient selfmanagement of chronic disease in primary care. JAMA. 2002;288(19):2469-75.

35. Miller CJ, Grogan-Kaylor A, Perron BE, Kilbourne AM, Woltmann E, Bauer MS. Collaborative chronic care models for mental health conditions: cumulative meta-analysis and metaregression to guide future research and implementation. Med Care. 2013;51(10):922-30.

36. Williams JW Jr, Gerrity M, Holsinger T, Dobscha S, Gaynes B, Dietrich A. Systematic review of multifaceted interventions to improve depression care. Gen Hosp Psychiatry. 2007;29(2):91-116.

37. Swift JK, Greenberg RP. Premature discontinuation in adult psychotherapy: a meta-analysis. J Consult Clin Psychol. 2012;80(4):547-59. 